\title{
On Necessity of Semantic Analysis when Evaluating Concept Maps
}

\author{
Maija Strautmane \\ Riga Technical University, Latvia
}

\begin{abstract}
Modern technologies have changed the landscape of teaching and learning in many ways, including introduction of computational support for knowledge assessment. Concept maps (CMs) can be used to externalise student's internal knowledge structure in a form suitable for computer-aided assessment. Current CM-based knowledge assessment systems do not analyse semantics of CM elements. The paper represents a study designed to find out how big is the portion of a CM that is left unevaluated in default of semantic analysis.
\end{abstract}

Keywords - Automated knowledge assessment, concept map, linking phrase, semantic analysis

\section{INTRODUCTION}

Advances in information and communication technology (ICT) have changed nearly every process in our everyday life; they have given new opportunities as well as challenges. In the field of teaching and learning, ICT can be used to enhance the study process in many ways, including automated knowledge assessment (AKA). AKA performs knowledge assessment asking minimal input from the tutor. The idea of a system that could provide a means for regularly and reliably assessing knowledge for large groups of students without significantly increasing the workload of a tutor is very appealing. It would allow giving feedback to students in a timely fashion and adjusting the teaching process in order to fill the gap and clear possible misunderstanding.

Internal knowledge structures are inaccessible directly for current knowledge assessment techniques [1]; therefore, student's knowledge structure should be externalised in some form, and concept maps (CMs) can be used for this purpose. CMs are graphical tools for organising and representing knowledge [2]. CM is a structure where concepts are depicted as vertices, and connecting lines between them correspond to their relationships. In their classical sense, CMs contain labelled lines that have a direction. A label explains the nature of relationship between concepts, while direction shows how this knowledge unit should be "read". Two concepts that are connected by a relationship form a proposition - a sentence-

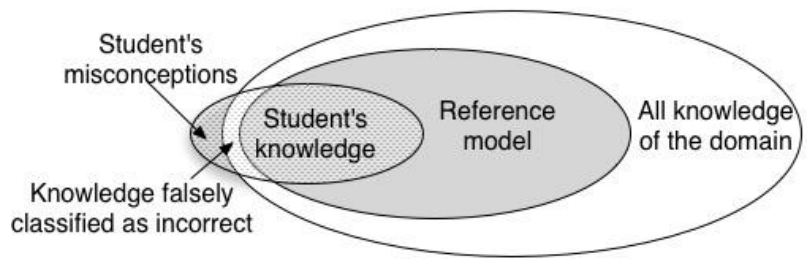

Fig. 1. The relationship between student's knowledge, reference model and all correct knowledge of the field. like structure that is a meaningful statement about some object or an event in the universe, either naturally occurring or constructed [2].

CMs can be and are used for both technology enhanced and manual knowledge assessment. The res emblance between CMs and graphs allows for calculation of various structural measures that can be used to characterise the quality of student's knowledge structure (see, for example, [3] and [4]). Another way to evaluate CMs is to use measures that evaluate the quality of CM components. Some examples of these measures are quality of concept labels, proposition's correctness or depth of explanation (for more information on CM measures, see [5]).

Those measures that can be calculated with little effort, such as a number of concepts and relationships, provide little insight into student's understanding [6], but those regarding the quality of $\mathrm{CM}$ elements ask for natural language processing. There is still no widely accepted method for scoring concept maps despite numerous attempts to create one (see, for example, [4], [7] or [8]).

When evaluating the quality of CM components with an automated knowledge assessment system (AKAS), it needs some reference model or a benchmark to compare every student's CM (see Fig.1). Although such an approach contradicts the constructivist postulate that no two people have the same mental schema [9], and their externalised knowledge can differ, but still represent correct knowledge of the field, currently no better way exists. In CM-based assessment, a reference model is a $\mathrm{CM}$ created by one or more experts and/or teachers. Reference models do not contain all knowledge on the subject. Instead they contain only the most relevant facts according to their creators' opinion. As CMs use a natural language to label relationships, another problem arises: different linking phrases can be used to describe a relationship in student's CM and in the reference model. The mentioned aspects lead to the situation where part of correct knowledge is classified as false negatives. It is partially due to the inability of assessment mechanisms to evaluate semantics of linking phrases. The term "semantic analysis" here refers to the examination of linking phrases used by student to determine whether they describe correct pieces of knowledge on the subject or not. As the semantic analysis of relationships is not an easy task and it would take a lot of effort to implement a system capable of such in-depth examination, the question arises: how large is the portion of a CM that is left unevaluated due to this flaw? The aim is not to prove whether or not semantic analysis would allow more precise assessment 
of a CM because it is clear that it would. The goal is to find out if the amount of propositions inaccessible for an AKA is large enough to countervail the work needed to implement an assessment mechanism that could analyze linking phrases.

This paper presents a study of $186 \mathrm{CMs}$ that were compared to a reference model and in each CM four classes of propositions were counted: matching ones, propositions without a label, ones that use a different label and those that are not present in the reference model. It was discovered that only about one fifth of propositions in studied CMs used the same linking phrases as in the reference model. In the second part of the study three propositions were examined in more detail to reveal how diverse are the used linking phrases and if all non-matching labels convey a completely different meaning or not. It was revealed that the variety of labels is rather wide and for two out of three propositions nonmatching linking phrases with a semantically close meaning were used even more frequently than matching ones.

Types of CM-based tasks are discussed in the second section, along with their scoring criteria and the challenges of CM-based AKA. The procedure of study is described in Section III and its results are demonstrated in Section IV. Conclusions and possible further studies are set forth in the Section V.

\section{CM-BASED KNOWLEDGE ASSESSMENT}

As stated in [4], AKA, in general, has several advantages when compared to manual labour-intensive methods: it allows working with large groups of students and from a methodological viewpoint may raise objectivity. AKA also enables a regular assessment that is important for giving students timely feedback about their progress. A tutor can use information from AKA about poorly understood themes and concepts to adjust teaching.

There have been numerous attempts to design AKASs that use CM-based tasks. According to [10], CM-based assessment consists of three parts: a task, response format and scoring system. Task consists of the given information and required information that a student has to provide to complete it. Response format means the media used for completing the task, and the scoring system describes what traits of a CM are evaluated and how important they are. All three parts of CMbased assessment are somewhat related. For example, both the type of task given and response format determine the choice of scoring system that can be used to evaluate CMs.

\section{A. CM-based Knowledge Assessment Tasks}

There are several aspects that make up a CM-based task. Many kinds of possible CM-based tasks can be created depending on whether the structure of a CM, linking phrases, and concept labels are given fully, partially or have to be provided by a student. According to [10], all CM-based tasks can be placed on the directedness axis starting from highdirected tasks to low directed ones. Directedness is influenced not only by what is provided, but also by the extent/amount of what is provided, the significance of what is provided and what is required from the examinees.
Two main types of tasks are "construct-a-map" (CCM) tasks where a student has to build a structure with or without given concepts and/or linking phrases and "fill-in-the-map" (FCM) tasks where a student has to complete the given structure by placing concepts and/or linking phrases in appropriate places. CCM tasks are generally considered lowdirected, while FCM tasks are high-directed. In [5], it was calculated that more than 700 types of tasks were possible, but only seven of them were used frequently, and around $85 \%$ of summarised documents on the subject reported the usage of CCM tasks without a given list of relationship labels.

When using CMs for knowledge assessment, it is important to choose such CM-based tasks that allow students to externalise their internal knowledge structures as precisely as possible. The Panamian example (see [11]) proves that widespread use of concept maps as a knowledge representation and assessment tool is not enough to impact the quality of education if they are used in the "wrong" way. The philosophy behind CMs is that meaningful learning gives better results than rote learning and thus should be encouraged. Results of meaningful learning are best observed when using less restricted types of CM-based tasks. Authors of [12] even believe that the FCM tasks should be regarded as inappropriate for measuring a student's knowledge structure because tasks of this kind too severely restrict the representation.

In high-directed CM-based tasks, a student's response is severely restricted by the given information, while lowdirected tasks give a student more freedom and let him/her more precisely express his/her knowledge structure [13]. The concept of the CM-based task directedness is further discussed in [14].

Self-generated knowledge structures are closest to contemporary views of constructivism [15]. Difficulties of using CMs result primarily from years of rote-mode learning practice in school settings [2]; thus, the use of CCM tasks would also foster meaningful learning instead of rote learning and allow differentiating between those two. Less constrained tasks also provide more insight into students' partial knowledge [6].

There are also several drawbacks of CCM tasks. The maximum number of propositions is uncertain [16], and more propositions does not always mean better knowledge on subject, it could also mean inability to differentiate between what is important and what are marginal pieces of knowledge. CCM tasks are considered an open-ended response because as each mental model has some unique components [17], it is not feasible to list all possible correct constructs in advance. AKASs are implemented only for about $41 \%$ of systems that use an open-ended response [18].

\section{B. Importance of Linking Phrases}

When choosing tasks for knowledge elicitation one also needs to consider, whether or not to provide lists of concepts and/or relationships. Although linking phrases are an essential part of a CM, providing a linking phrase is the hardest task when constructing a CM [2]. Difficulties to label relationships 
mean that a student poorly understands the relationship between the concepts or the meanings of the concepts [2]. CCM tasks with the provided list of concept labels allow automatic assessment, but they also elicit fewer propositions than tasks without lists [16]. The wide usage of CCM tasks without lists of linking phrases means that the scoring system that simply matches the student's created labelled links with the ones in reference model would only be partially able to assess the quality of relationships in the student's CM as even the addition or omission of a preposition or ending creates a different linking phrase.

As the evaluation of linking phrases takes a lot of effort, it could seem tempting to take into consideration only the existence of a relationship, but in the previous study [19] the author of this paper has proven that a linking phrase gives such information about student's knowledge that cannot be obtained by only taking into account the presence of a link. The in-depth discussion on criteria that could be used for automated assessment is provided in the next section.

\section{CM Evaluation Criteria}

In [5], an overview of criteria used for the CM assessment has been published, and not many of reported criteria are calculated automatically. The automation of structural measures is less problematic. Authors of [20] state that as CM main building blocks are the structural elements, it is technically feasible to develop a formal computational model to estimate the quality of the concept map based on its structural elements.

There are quite a few parameters of CMs that can be calculated automatically without teacher's input in the process, such as a diameter of a CM, number of ingoing and

outgoing links, depth and breadth of a CM, density (relationships per concept) and many others (see [5]). However, it seems that attempts to use various combinations of structural criteria for CM assessment have not been fruitful mainly because they lack pedagogical ground. It is not clear what exactly these criteria (by themselves and in various combinations) reveal about the knowledge structure of the CM creator. There are some attempts, for example, [1], [20] and [21] to define what certain criteria measure in terms of the underlying knowledge structure, but it is still not clear what values represent knowledge structure of good quality and what values bear witness of poor knowledge. For example, in [20], it is stated that a narrower and deeper knowledge structure implies that its creator focuses more on deep thinking that contributes toward the development of solutions to a particular problem. At the same time, in [22] the possessor of deep and narrow knowledge structures (chains) is described unable to use the knowledge in new situations and different contexts. Therefore, it is not clear whether having a high value of the depth of a structure is a positive or a negative trait. The depth or the breadth of a knowledge structure also depends on the subject that is considered, but the point is that these criteria are not unambiguous. To the author's knowledge, there are not reports of successful long-term usage of structural criteria without estimating the quality of CM elements.

Authors of [12] state that a concept is defined by its relation to other concepts; thus, to determine how well a student understands particular concepts it would be meaningful to analyse relationships between concepts. As described previously, a linking phrase is an important part of a relationship; thus, the analysis of a $\mathrm{CM}$ would have to take into consideration also this component of proposition.

In fact, the quality of propositions is considered to be one of the most important criteria [23] - the quality of propositions determines the quality of a CM as a whole [24]. At the same time, this is one of the most problematic criteria when considering its automation. Current AKAS like IKAS [7], SMD technology [4] are only able to count linking phrases as correct if they exactly match a reference model. IKAS also supports manual augmentation of a reference model by adding synonyms to of linking phrases so one link could have more than one correct label. Nevertheless this system also is able to recognise as correct only the phrases that exactly match the reference model. To more precisely evaluate the quality of each proposition, it would take a lot of effort in terms of semantic analysis. According to [25] in CMs, both concepts and propositions are represented in a natural language and, as a result, are subject to ambiguity. Therefore, the initial enthusiasm about advantages that the use of CMs would give compared to other knowledge assessment methods has cooled off.

The semantic analysis is necessary for evaluation of relationships described with linking phrases other than in the reference model as well as for relationships that connect concepts unrelated in the reference model. The fact that two concepts are unrelated in the reference model does not mean that there is no connection between them. In fact, everything in the universe is connected in some way; thus, links without labels do not mean much [26]. As reference models contain a refined set of relations and concepts and not every single possible proposition that represents correct knowledge in the domain student's created structures may also be correct even if they do not appear in the reference model.

It is hypothesised that for CCM tasks without a given list of linking phrases a great majority of propositions are classified as incorrect when, in fact, they could also be correct or partially correct. Thus, a study has been designed to answer the question whether or not it is a great part of CM that is left unrecognised by an assessment mechanism that is only able to spot exact matches. 


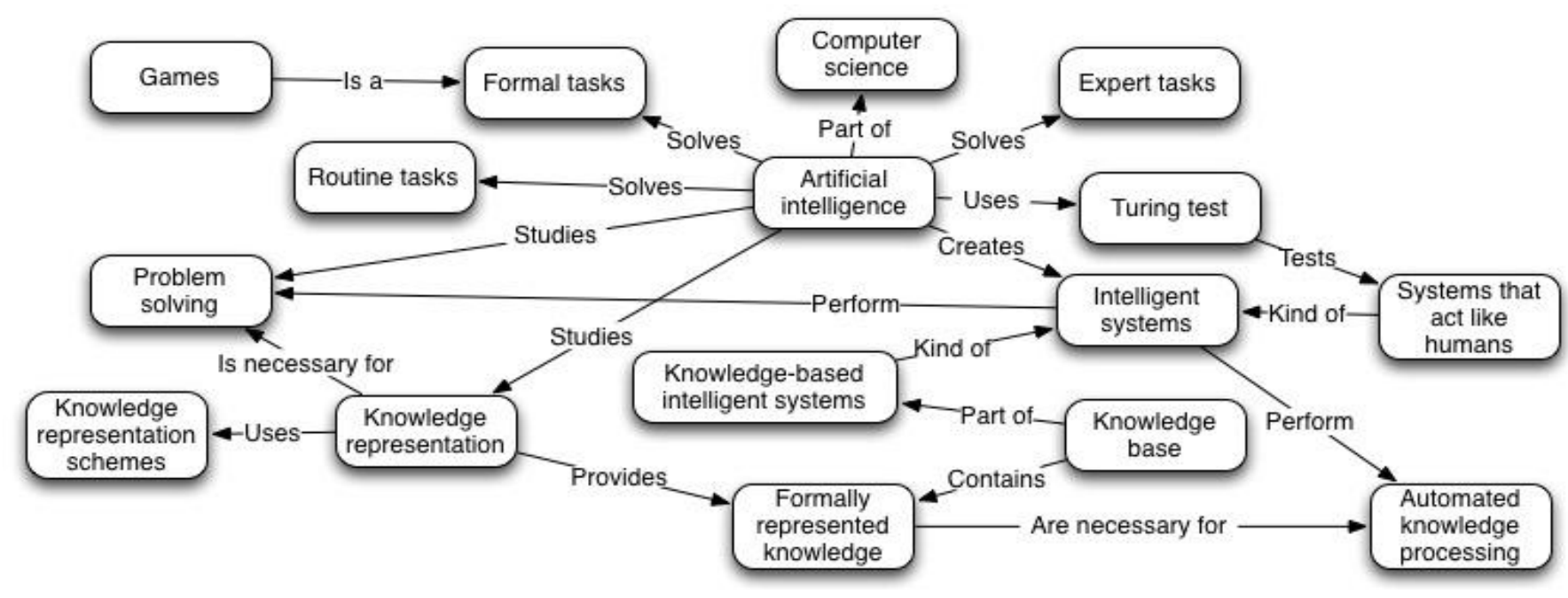

Fig. 2. Part of a reference model used for the study

\section{STUDY}

To conduct this study, the author used $186 \mathrm{CMs}$ from the previous study reported in [19] where it was proven that a linking phrase is an essential part of a proposition and should be taken into consideration when evaluating knowledge represented as CMs. The authors of these CMs were third-year bachelor's programme students at Riga Technical University, Faculty of Computer Science and Information Technology that had the course "Fundamentals of Artificial Intelligence" in the spring semester of the year 2013. All of them had some previous experience with CMs so no additional training was necessary. As students could gain partial credit for participating in this study (up to $10 \%$ of the final grade depending on the quality of submitted CM) they were interested in creating as qualitative $\mathrm{CM}$ as possible.

The CM-based task that was used for this study was a construct-a-map task with a given list of concepts that contained 41 concepts from the study course. In the task statement, participants were reminded about such frequently used linking phrases as "is-a", "part-of", "characterises", "is example" and "kind of". They were also encouraged to use any other linking phrase that they find appropriate for describing the particular relationship between concepts. Each participant received a sheet of A3 paper and had to complete the task in 90 minutes.

As a reference model, a CM cooperatively created by the author of the paper and the tutor of the mentioned course was used in the study. It contained 55 relationships that were considered to represent important knowledge in the domain (see Fig. 2. for fragment of this CM).

In each student's CM, such relationships were counted manually: (a) relationships identical to reference model, (b) relationships without any linking phrase, (c) relationships labelled with a different linking phrase than in a reference model, and (d) relationships absent in a reference model. There was no upper limit to how many links to include in the $\mathrm{CM}$, and the author was more interested in percentage than absolute numbers; thus, for each of these four classes of relationships the ratios were calculated dividing the number of relations in a particular class by the total number of relations in the CM.

For each of classes (c) and (d), a different strategy involving the semantic analysis could be used to make them accessible for AKAS. These two classes together make up the portion of a $\mathrm{CM}$ that is inaccessible to the current $\mathrm{CM}$ assessment algorithms; thus, it would be informative to calculate how big part of CMs they comprise. This would motivate the decision to design and implement an AKAS with the semantic analysis of relationships or not.

After the above-mentioned calculations, it was decided to take a closer look on how diverse the linking phrases were for three propositions (see Fig. 3). These three propositions were chosen because they were present in about half or more than half of students' CMs and the variety of linking phrases used for describing the nature of each of them was rather wide. The results of this investigation would serve as

a basis for further studies on semantic analysis mechanisms that could be used for CM-based AKA.

As students completed their CMs in Latvian, linking phrases were also expressed in this language, and here all of them would not be mentioned as the translation would in some cases slightly change the meaning and make ungrammatical constructs in English. Instead, the paper presents a summary of how many diverse linking phrases were used and how many

(a)

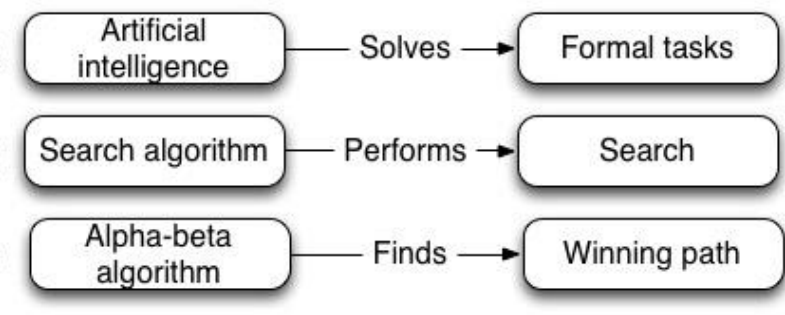

Fig. 3. Propositions used for study 
students used linking phrases that were close in their meaning to the ones used in the reference model. The number of exact matches is presented as well. The amount of semantically close propositions was counted because the strategy for evaluating these cases could be different and possibly easier to implement than cases where a linking phrase had a different meaning. It was the author of this paper who determined whether two linking phrases were semantically close or not.

The fact that a linking phrase is not semantically close or exactly matching the one used in the reference model does not mean that it is incorrect because there is more than only one relationship possible between two concepts [27].

\section{RESULTS}

For each class of relationships a maximum percentage, a minimum percentage and a median were calculated (see Table I). Median values were used instead of mean values to avoid being affected by extreme values. Values of standard deviations were also calculated to obtain the normal range of values for each class of relationships. As there were no limitations of how many propositions to include in a CM, the total number of relationships in students' created CMs ranged from 22 to 59 with average being 39.46. That was $30 \%$ less than in the reference model. The fact that there were fewer relationships in students' CMs than in the reference model showed that not all students had mastered all the concepts equally well. This is a standard situation because the author of the paper did not select only the most successful students for this study, and capabilities and the amount of work invested in studies were not the same for all students. On average the CM created by a student consisted of about $21 \%$ propositions that exactly matched the reference model, about $34 \%$ propositions that were described using other linking phrase than in the reference model, and about $38 \%$ propositions that connected concepts that were not connected in the reference model. Most of the student's CMs contained no unlabelled relationships although there were students that were not able to provide linking phrases for up to $64 \%$ of all relationships they created. For each class, a chart was created to visually depict the range of ratios (see Fig. 4). Lines were added to these charts to show
TABLE I

PERCENTAGE OF DifFERENT Classes of RELATIONSHIPS

\begin{tabular}{|l|l|l|l|l|}
\hline & $\begin{array}{l}\text { Exact } \\
\text { Match }\end{array}$ & $\begin{array}{l}\text { Without } \\
\text { Phrase }\end{array}$ & $\begin{array}{l}\text { Other } \\
\text { Phrase }\end{array}$ & $\begin{array}{l}\text { Not in the } \\
\text { reference } \\
\text { model }\end{array}$ \\
\hline $\begin{array}{l}\text { Min } \\
\text { percentage }\end{array}$ & 0 & 0 & 0 & 0 \\
\hline $\begin{array}{l}\text { Max } \\
\text { percentage }\end{array}$ & 70 & 64 & 83.33 & 96 \\
\hline $\begin{array}{l}\text { Median } \\
\text { percentage }\end{array}$ & 21.74 & 0 & 34.09 & 38.24 \\
\hline SD & 13.24 & 11.43 & 15.03 & 14.51 \\
\hline
\end{tabular}

TABLE II

Number of EXactly Matching AND Semantically Close Propositions

\begin{tabular}{|c|c|c|c|}
\hline & 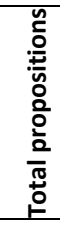 & 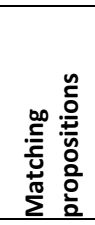 & 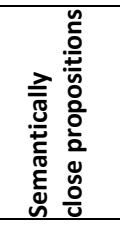 \\
\hline $\begin{array}{l}\text { (a)[Artificial Intelligence] } \\
\text {--Solves--> [Formal tasks] }\end{array}$ & 102 & $\begin{array}{l}25 \\
(25 \%)\end{array}$ & $\begin{array}{l}18 \\
(18 \%)\end{array}$ \\
\hline $\begin{array}{l}\text { (B)[Search algorithm] } \\
-- \text { Performs--> [Search] }\end{array}$ & 139 & $5(4 \%)$ & $54(39 \%)$ \\
\hline $\begin{array}{l}\text { (C)[Alpha-beta algorithm] } \\
\text {-Finds--> [Winning path] }\end{array}$ & 84 & $5(6 \%)$ & $29(35 \%)$ \\
\hline
\end{tabular}

TABLE III

NUMBER OF DIFFERENT LINKING PHRASES USED FOR PROPOSITIONS

\begin{tabular}{|l|l|l|}
\hline & $\begin{array}{l}\text { Number of } \\
\text { different } \\
\text { linking phrases }\end{array}$ & $\begin{array}{l}\text { Number of } \\
\text { linking phrases } \\
\text { used more than } \\
\text { once }\end{array}$ \\
\hline $\begin{array}{l}\text { [Artificial Intelligence]-Solves-- > } \\
\text { [Formal tasks] }\end{array}$ & 25 & 12 \\
\hline $\begin{array}{l}\text { [Search algorithm]-Performs--> } \\
\text { [Search] }\end{array}$ & 40 & 23 \\
\hline $\begin{array}{l}\text { [Alpha-beta algorithm]-Finds--> } \\
\text { [Winning path] }\end{array}$ & 18 & 12 \\
\hline
\end{tabular}

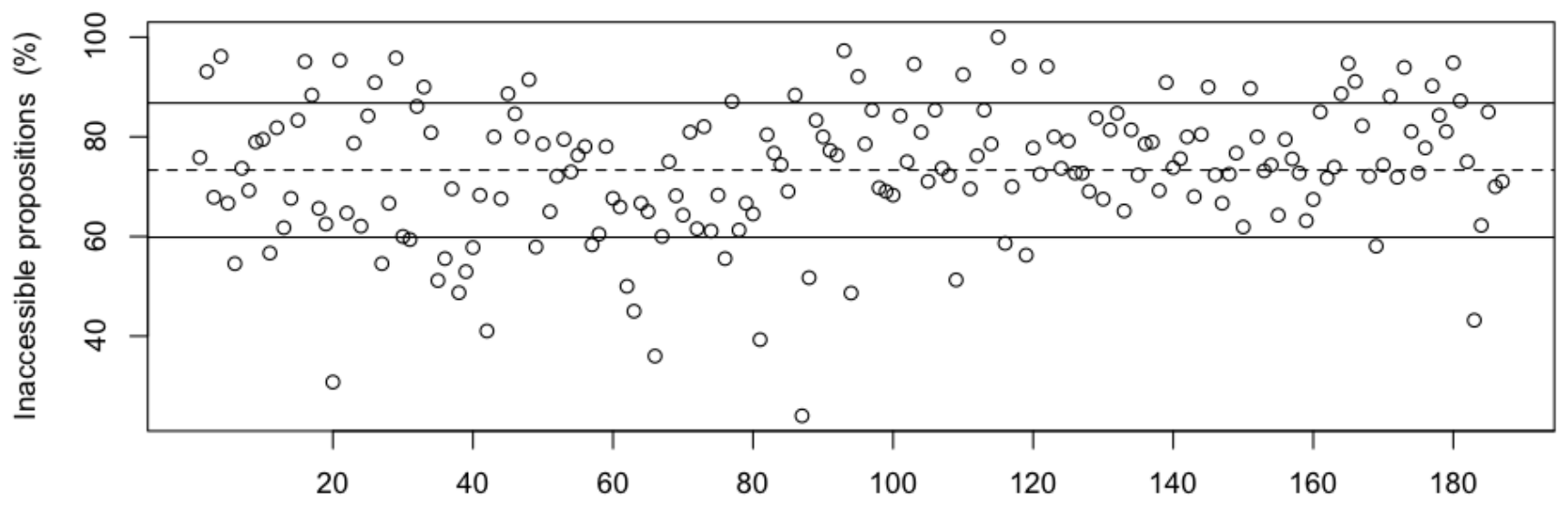

Index of a participant's CM

Fig. 4. Ratios of propositions of each class to the total number of propositions in students' CMs 


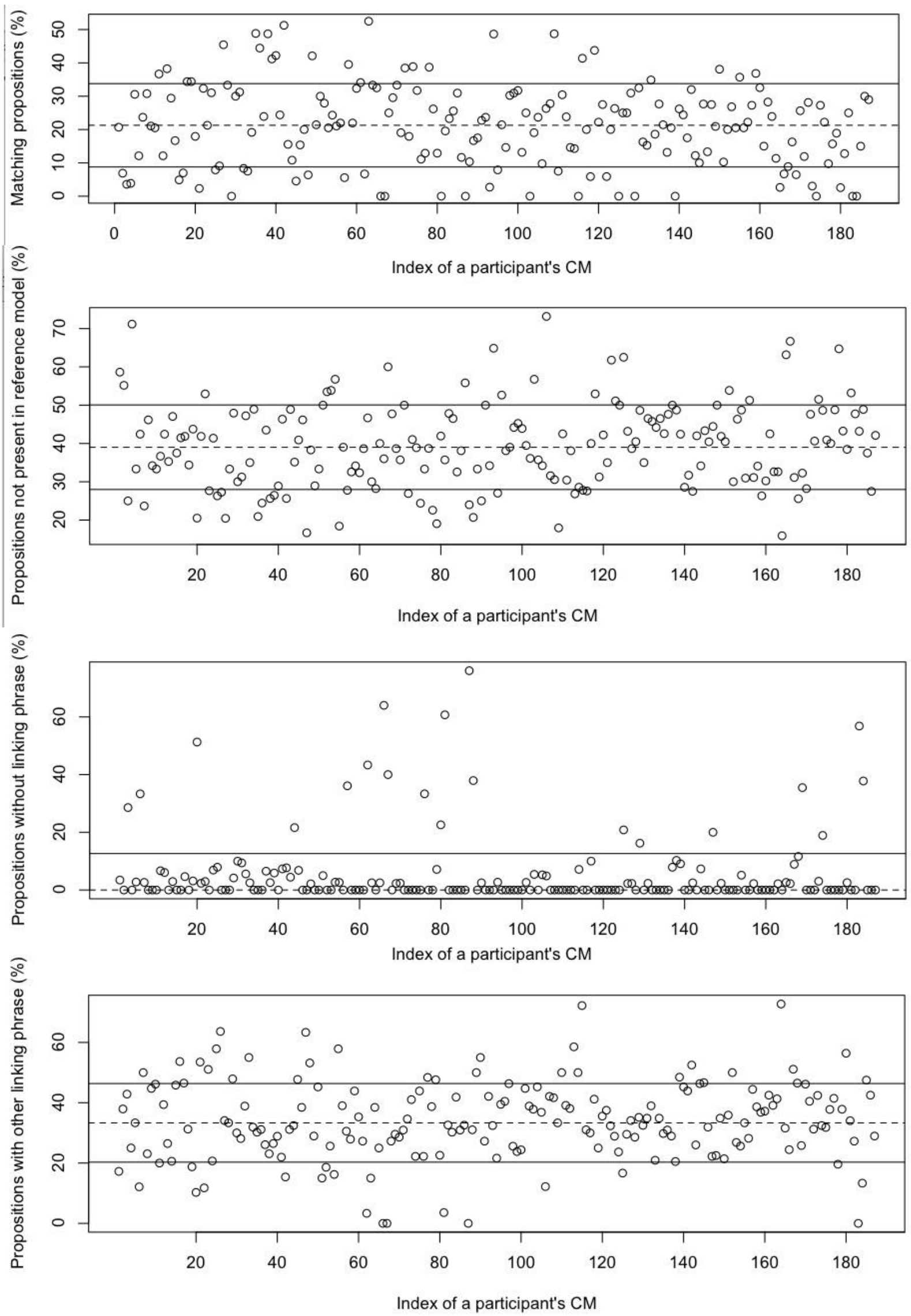

Fig. 5. Ratio of inaccessible propositions to the total number of propositions in students' CMs 
a median value and a standard deviation. As can be seen from these charts, most participants were able to construct from $9 \%$ to $35 \%$ propositions that exactly matched the ones in the reference model, from $19 \%$ to $49 \%$ propositions that connected the same concepts as in the reference model but used different linking phrases for describing the relationship, from $24 \%$ to $53 \%$ propositions that connected such concepts that were not connected in the reference model, and up to $11 \%$ links without linking phrases.

Analysing the ratio of inaccessible propositions (see Fig. 5.), i.e. those with other linking phrases or not existing in the reference model, it was discovered that on average $73 \%$ (normally ranging from $60 \%$ to $87 \%$ ) of propositions would be classified as incorrect by AKAS that were able only to differentiate between exact matches and other linking phrases.

When analysing linking phrases used for describing propositions that were included in the second part of the study, it was noted that not all CMs contained a link between respective two concepts. Proposition (c) was found in less than half (84) of all participants' CMs. Other two propositions were present in 102 and 139 CMs, respectively. In Table II, results of the second part of the study are presented. It can be observed that for the chosen three propositions students used exactly the same linking phrase as in the reference model quite rarely: about $25 \%$ of the times for proposition (a) and $4 \%$ and $6 \%$ for propositions (b) and (c), respectively.

The situation is notably different when calculating the number of semantically close propositions. In the present paper, semantically close propositions are defined all those propositions that use almost the same linking phrase with only slight differences as well as those that use different words for describing a similar relationship between concepts.. For example, phrases "searches", "finds", "determines", "allows determining", and "derives" for the proposition (c) are all considered conveying a semantically close meaning.

For the proposition (a) every fifth student used a different linking phrase that had similar meaning to the one used in the reference model. Numbers are even greater for propositions (b) and (c): $39 \%$ and $35 \%$, respectively. The meaning of these two propositions were put into words differently than in the reference model much more frequently than they were labelled with the same phrase. This corresponds to the opinion presented in [28] that different people will most likely map the same topic differently. From these results, it is visible that the portion of propositions that an AKAS would recognise as correct could significantly increase if only it had ability to determinate whether or not two linking phrases described the same underlying meaning.

If the numbers of last two columns of Table II are added, the number of students trying to express a relationship similar to the one present in the reference model is obtained. Interestingly, sums of these numbers are quite alike for all three propositions (around 41-43\%). This is an interesting tendency, but it is not possible to generalise these results due to a small sample size. Nevertheless, it shows that despite using different linking phrases, little less than half of the participants grasped the meaning that their tutor tried to teach them. Thus, the CM scoring mechanism should be able to recognise these propositions as representing correct knowledge.

The variety of linking phrases (including the exactly matching phrase) used for each of the three chosen propositions is quite high (see Table III): 25 linking phrases for the proposition (a) and 40 and 18 for propositions (b) and (c), respectively. Not all of them would be considered as correctly describing the nature of the relationship. Some of them were describing partially correct propositions or even completely erroneous assumptions. As many of the linking phrases were used only by one student, the author also counted how many of them were present in more than one CM. These numbers are still high and thus the results of the second part of the study also support the hypothesis that a CM-based AKAS would benefit from the ability to analyse the meaning behind a linking phrase.

\section{CONCLUSIONS AND FURTHER RESEARCH}

The study has proven that for CM construction tasks where students themselves provide labels for linking lines, simple matching of linking phrases is able to evaluate only a little more than $20 \%$ of propositions. As such tasks are used most frequently for knowledge assessment, and the responses to them are considered to better represent student's knowledge, it would be useful to implement mechanisms that perform some kind of semantic analysis to evaluate the quality of propositions in order to assess a CM to its full extent.

The number of propositions that are inaccessible for AKAS is far greater than expected considering the fact that the task with the given list of concepts was employed and students could use their study materials during the construction of their CMs. Thus, it can be concluded that the ability of AKASs to evaluate the quality of propositions and thus of the CM as a whole would significantly increase by using some natural language processing.

The second part of the study shows that a majority of dissimilar linking phrases are semantically close to those used in the reference model; thus, a mechanism that would allow identifying these alternative linking phrases would make it possible to further reduce the portion of $\mathrm{CMs}$ currently inaccessible for AKA.

It has been observed during the second part of the study that in cases where linking phrases do not match the reference model it is not that linking phrases used for explaining the nature of relationships would always use completely different words. Even an added or omitted preposition, an ending of a verb or noun and/or an added or omitted auxiliary verb can make two linking phrases non-matching even if it does not significantly change semantics of a proposition. This part of the study has proven that the number of propositions that are considered non-matching but are conveying the same meaning as in the reference model is considerably large.

The results of the second part of the study cannot be generalised, as only three propositions were analysed. However, it is clear that the number of inaccessible 
propositions could be decreased by a mechanism that could generate all the linking phrases making semantically similar propositions and add them to the reference model as possible substitutes for the phrase used there. The wide variety of linking phrases used by students for describing each of the propositions proves that it would be a very time-consuming task to predict all possible correct linking phrases and add them to the reference model in advance without the help of an automated system.

There is a demand for knowledge assessment tools that could be used for e-learning and for large groups of students. CM-based AKAS could be one of the options that alleviate the workload of a tutor by using ICT. Further research on how to improve CM-based AKA could take either or both of these two directions: (1) how to automatically augment a reference model so that it becomes more complete and (2) how to automatically analyse semantics of relationships using natural language processing techniques. The first direction also involves the creation of the mechanism that could determine possible linking phrases for semantically close propositions. To the author's opinion, it is necessary to classify the cases how two propositions can differ because it seems that not all of them could be solved with the same mechanism.

\section{ACKNOWLEDGEMENT}

The author is thankful to her colleagues then assistant professor now associate professor Alla Anohina-Naumeca and professor Jānis Grundspenkis who taught the course "Fundamentals of Artificial Intelligence" in the spring semester of 2013 for letting her conduct the study presented here.

\section{REFERENCES}

[1] D. Ifenthaler, "Scope of Graphical Indices in Educational Diagnostics," in Computer-Based Diagnostics and Systematic Analysis of Knowledge, D. Ifenthaler, P. Pirnay-Dummer, and N. M. Seel Eds. New York, NY Springer, 2010, pp. 213-234. https://doi.org/10.1007/978-1-4419-5662$\underline{0 \_12}$

[2] J. D. Novak and A. J. Canas, "The Theory Underlying Concept Maps and How to Construct and Use Them," 2006. [Online]. Available: http://cmap.ihmc.us/publications/researchpapers/theorycmaps/theoryund erlyingconceptmaps.htm [Accessed: Apr. 27, 2017].

[3] A. J. Cañas, L. Bunch, J. D. Novak, and P. Reiska, "Cmapanalysis: an extensible concept map analysis tool," Journal for Educators, Teachers and Trainers. vol. 4, no. 1 (Special), pp. 36-46, 2013.

[4] [4] P. Pirnay-Dummer and D. Ifenthaler, "Automated knowledge visualization and assessment," in Computer-based diagnostics and systematic analysis of knowledge, D. Ifenthaler, P. Pirnay-Dummer, and N. M. Seel, Eds. New York: Springer, 2010, pp. 77-115. https://doi.org/10.1007/978-1-4419-5662-0 6

[5] M. Strautmane, "Concept Map-Based Knowledge Assessment Tasks and Their Scoring Criteria: an Overview," in Concept Maps: Theory Methodology, Technology: Proceedings of the Fifth International Conference on Concept Mapping, Malta, Valletta, 2012, pp. 80-89.

[6] B. A. Schwendimann, "Making sense of Knowledge Integration Maps," in Digital knowledge maps in education: Technology enhanced support for teachers and learners D. Ifenthaler and R. Hanewald, Eds. Springer, 2014, pp. 17-40. https://doi.org/10.1007/978-1-4614-3178-7_2

[7] A. Anohina-Naumeca, J. Grundspenkis, and M. Strautmane, "The Concept Map Based Assessment System: Functional Capabilities, Evolution, and Experimental Results," International Journal of Continuing Engineering Education and Life-Long Learning, vol. 21, no. 4, pp. 308-327, 2011. https://doi.org/10.1504/ijceell.2011.042790
[8] B. E. Cline, C. C. Brewster, R. D. Fell "A rule-based system for automatically evaluating student concept maps." Expert systems with $\begin{array}{lllll}\text { applications, } & \text { vol } \quad 37, \quad \text { pp. } & 2282-2291, & 2010\end{array}$ https://doi.org/10.1016/j.eswa.2009.07.044

[9] D. A. Lapp, M. A. Nyman, and L. S. Berry, "Student connections of linear algebra concepts: an analysis of concept maps," International Journal of Mathematical Education in Science and Technology, vol. 41, no. 1, pp. 1-18, 2010. https://doi.org/10.1080/00207390903236665

[10] M. A. Ruiz-Primo, "Examining Concept Maps as an Assessment Tool," in Concept Maps: Theory, Methodology, Technology. Proceedings of the First International Conference on Concept Mapping, A. J. Cañas, J. D. Novak, and F. M. González, Eds. Pamplona, Spain 2004, pp. 555-562.

[11] N. Miller, A. J. Cañas, and J. D. Novak, "Preconceptions Regarding Concept Maps Held by Panamanian Teachers," in Concept Maps: Theory, Methodology, Technology Proceedings of the Second International Conference on Concept Mapping A. J. Cañas, and J. D. Novak, Eds. San José, Costa Rica, 2006, pp. 469-476.

[12] M. A. Ruiz-Primo and R. J. Shavelson, "Problems and Issues in the Use of Concept Maps in Science Assessment," Journal of Research in Science Teaching, vol. 33, no. 6, pp. 569-600, 1996. https://doi.org/10.1002/(sici)1098-2736(199608)33:6<569::aidtea1>3.0.co;2-m

[13] B. A. Schwendimann, "Mapping Biological ideas: concept maps as knowledge integration tools for evolution education," Doctoral thesis, University of California, CA USA, 2011, p. 382.

[14] A. Anohina-Naumeca and V. Graudina, "Diversity of concept mapping tasks: degree of difficulty, directedness, and task constraints," in Concept Maps: Theory, Methodology, Technology. Proceedings of the Fifth International Conference on Concept Mapping, vol. 1, A. J. Canas, J. D. Novak, and J. Vanhear, Eds., Valleta, Malta, 2012, pp. 164-171.

[15] A. M. O'Donnell, D. F. Dansereau, and R. H. Hall, "Knowledge Maps as Scaffolds for Cognitive Processing," Educational Psychology Review, vol. 14, no. 1, pp. 71-86, 2002. https://doi.org/10.1023/A:1013132527007

[16] Y. Yin, J. Vanides, M. A. Ruiz-Primo, C. C. Ayala, and R. J. Shavelson, "Comparison of Two Concept-Mapping Techniques: Implications for Scoring, Interpretation, and Use," Journal of Research in Science Teaching, vol. 42, no. 2, pp. 166-184, 2005. https://doi.org/10.1002/tea.20049

[17] C. Schau, N. Mattern, R. J. Weber, K. Minnick, and C. Witt, "Use of fill-in concept maps to assess middle school students connected understanding of science," Paper presented at the Annual Meeting of the American Educational Research Association, Chicago, IL, USA, 1997.

[18] C.-Y. Kuo and H.-K. Wu, "Toward an integrated model for designing assessment systems: An analysis of the current status of computer-based assessments in science," Computers \& Education, vol. 68, pp. 388-403, 2013. https://doi.org/10.1016/j.compedu.2013.06.002

[19] M. Strautmane, "Usage of Semantics as a Part of Links as the Basis for Learner's Knowledge Structure Assessment The Pros and Cons." in proceedings of ICEE/ICIT, 2014

[20] J.-X. Hao, R. C.-W. Kwok, R. Y.-K. Lau, and A. Y. Yu, "Predicting problem-solving performance with concept maps: an informationtheoretic approach," Decision support systems, vol. 48, pp. 613-621, Mar. 2010. https://doi.org/10.1016/j.dss.2009.12.001

[21] B. A. Greene, I. A. Lubin, J. L. Slater, and S. E. Walden, "Mapping Changes in science teachers' content knowledge: concept maps and authentic professional development," Journal of science Education and Technology, vol. 22, no. 3, pp. 287-299, 2012. https://doi.org/10.1007/s10956-012-9393-9

[22] D. B. Hay and I. M. Kinchin, "Using concept maps to reveal conceptual typologies," Education + Training, vol. 48, no. 2/3, pp. 127-142, Feb. 2006. https://doi.org/10.1108/00400910610651764

[23] Z. Raud, V. Vodovozov, and T. Lehtla, "Teaching, Learning, and Assessment Integration in Electronics on the Concept Map Basis," in Innovating with Concept Mapping Proceedings of the Seventh International Conference on Concept Mapping, Tallinn, Estonia 2016, pp. 199-207.

[24] G. Lammers and C. M. Brown, "Exploring Student Understanding of Parallelism Using Concept Maps," in 2012 Proceeding of Frontiers in Education Conference, Oct. 2012, pp. 1-5. https://doi.org/10.1109/fie.2012.6462511

[25] J. V. Jr. da Costa, F. E. L. da Rocha, and E. L. Favero, "Linking phrases in concept maps: a study on the nature of inclusivity," in Proceedings of 
the First International Conference on Concept Mapping, A. J. Canas, J. D. Novak, F. M. Gonzalez, Eds. 2004.

[26] M. Ålberg, "Concept maps, mind maps and other similar graphic knowledge representation tools", invited manuscript to a Special Edition of Reflecting Education, vol. 3, 2007.

[27] R. J. Shavelson, H. Lang, and B. Lewin "On Concept Maps as Potential 'Authentic' Assessments in Science: Indirect Approaches to Knowledge Representation of High School Science". Los Angeles, CA: National Center for Research on Evaluation, Standards, and Student Testing, 1996. [Online]. Available: http://files.eric.ed.gov/fulltext/ED367691.pdf [Accessed: Apr. 27, 2017].

[28] B. Marshall, H. Chen, T Madhusudan, "Matching knowledge elements in concept maps using a similarity flooding algorithm," Decision Support Systems, vol. 42, no. 3, pp. 1290-1306, Dec. 2006. https://doi.org/10.1016/j.dss.2005.10.009

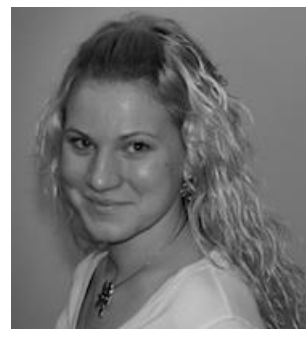

Maija Strautmane holds a BSc degree in computer control and computer science (since 2007) and an MSc degree in computer systems (since 2010) from Riga Technical University.

She has been working as a Laboratory Assistant and Research Assistant since 2006. Since 2010, she has been a Researcher at the Institute of Applied Computer Systems, Riga Technical University. M. Strautmane was a member of automated concept-map based knowledge assessment system IKAS development team in the period from 2007 to 2010. She has several publications concerning the usage of concept maps for automated knowledge assessment. Her main research interests include knowledge representation using concept maps and concept-map based automated knowledge assessment systems.

E-mail: maija.strautmane@rtu.lv 